\title{
ROBUST ADAPTIVE INTRA REFRESH FOR MULTIVIEW VIDEO
}

\author{
Sagir Lawan1 and Abdul H. Sadka2 \\ ${ }^{1 \text { and } 2}$ Department of Electronic and Computer Engineering, Brunel University, London, \\ UK
}

\begin{abstract}
Transmission error propagation in wireless multimedia communication systems has become a recurring problem. This persistent problem has led to grave consequences on the visual quality of the decoded video. It is against this backdrop that, we present an adaptive intra refresh (AIR) error-resilient coding tool to mitigate the effect of transmission error propagation in $3 D$ video communications. This work utilizes periodic insertion of intra macroblocks in badly error-infected frames temporally as well as related frames in the multi view video scheme. Our objective is to maximize the transmission efficiency while ensuring the transmission robustness of the coded bitstream. The selection of periodic macroblocks is based on areas with high motion above a pre-set threshold. The coding modes of the macroblocks are based on the distortion expectation due to transmission errors. Extensive simulation results show significant improvement in both objective and subjective video quality at different intra refresh rates.
\end{abstract}

\section{KEYWORDS}

Adaptive Intra Refresh, Multiview Video Coding, Error Resilience

\section{INTRODUCTION}

In recent years, there has been an increasing use of mobile communication devices in all sectors of human activities. Today, there is hardly a human activity in which there is no wireless multimedia communication utilized in one way or the other. Even warfighting concepts have equally evolved along these lines, as the battlefield of recent conflicts has been dominated by the force with the superior wireless multimedia information devices [1]. Wireless multimedia communications come in the form of text, images, sound, motion graphics, web sites, and video.

However, a major challenge in using wireless multimedia services, particularly video services, is the sensitivity of video signals to network errors. Errors such as bit and bust errors are preventing video signals from reaching their full potential. These transmission errors propagate among frames and views as illustrated in Figure 1. To ensure a 3D error resilient video transmission over error prone channels, a simple but efficient AIR method is proposed. This technique involves inserting intra macroblocks periodically in every nth frame and mth view that are dependent on the erroneous frame in order to mitigate the effect of error propagation.

Recent streams of published papers about video compression talk about how motion prediction reduces temporal redundancy. But, motion prediction introduces an error in a reference picture,

DOI : $10.5121 /$ ijcsea.2014.4601 
this error propagates both in space and time and affects the encoded reference picture. Encoding a number of motion active macroblocks with intra mode coding recovers the corrupted motion information. In [2]-[3]-[4], this idea of intra coding in the motion area defined the AIR error resilience technique. AIR works perfectly in pervious codecs (MPEG-4, H.263 etc), but, further research is required for its implementation in multiview video $\mathrm{H} .264$ codec, where only $\mathrm{P}$ or B pictures in a group of pictures (GOP) will be encoded.

This paper is organized as follows: Section 2 reviews the relevant literature on intra macroblock refresh scheme. The proposed AIR scheme for 3D is introduced in Section 3. Simulation setup and results are presented in Section 4, followed by conclusions in Section 5.

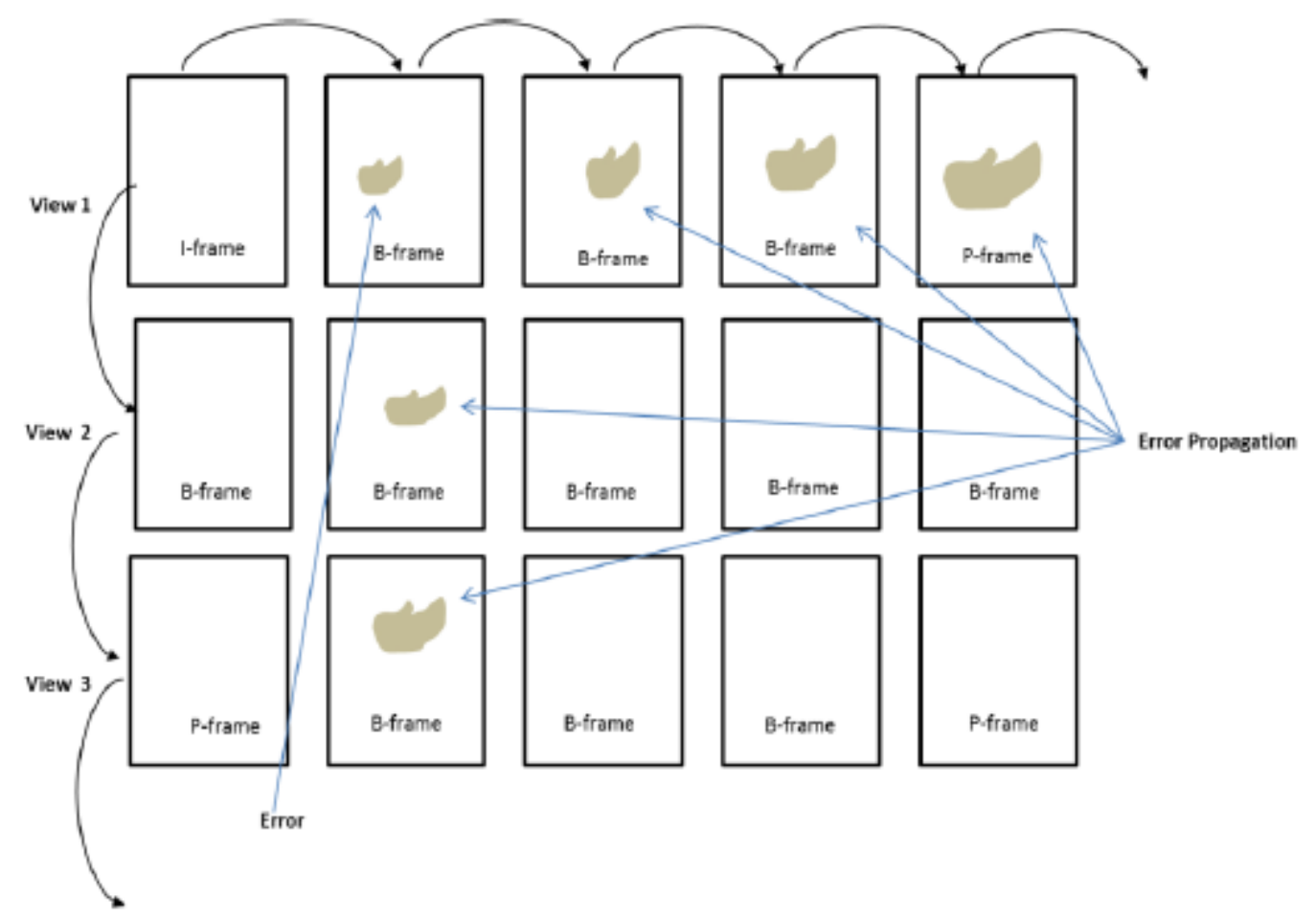

Figure 1. Error propagation in space, time and inter-view domains

\section{INTRA MACROBLOCK REFRESH SCHEME}

Several research activities addressed the Intra macroblock refresh schemes as an error resilience tool which can efficiently mitigate error propagation. The early intra refresh technique that emerged in history is the periodic insertion of intra macroblocks in a frame [5]. Thus, there are a number of various intra refresh techniques, which consequently changed the face of video transmission over the years. The most common technique is to refresh an entire frame with intra mode coding which means that the total frame encoding is to be in intra mode. However, this technique has a serious limitation as it results in a massive increase in data rate and hence this defeats the objective of compression. It also introduces a vast data which leads to serious errorfree quality degradation resulting from coarse quantisation losses [6] [7]. 
End-to-end rate distortion optimization proposed in [8] opened the door for random insertion of Ipictures. This new kind of intra refresh technique randomly refresh a frame sequence with fully Intra-coded picture (I-picture) inserted at regular intervals.

In the use of Intra refresh, considerable attention is paid to a feedback channel which is used to provide communication links between the far-end decoder and the source coder. Feedback channel conveys a notification message from the decoder requesting the transmission of an Iframe whenever it is required to recover from channel errors or else for zapping purposes [9]. In the absence of a feedback channel, the use of calculated macroblocks has a significant effect on the complexity of the system [10]. The motion impact of each macroblock is calculated and the macroblocks that have a significant motion activity are intra-coded. The ultimate goal of this method is to avoid the need for a feedback channel but at the expense of computational complexity.

\section{PROPOSED ADAPTIVE INTRA REFRESH METHOD FOR MULTIVIEW VIDEO CODING}

To aid understanding of the proposed AIR technique, two issues are discussed. The first is the generation of refresh map, and the second is the, implementation of AIR for the multi view scheme of H.264/AVC.

\subsection{Generation of Refresh Map}

In order to establish motion map, the proposed model exploits the correlation of MVC prediction. The temporal relationship between frames and the inter camera view relationship has been considered. In our solution, a GOP in Figure 2 is chosen to be Intra refreshed. To generate the Refresh Map, the following AIR step by step method is considered [11] [12].

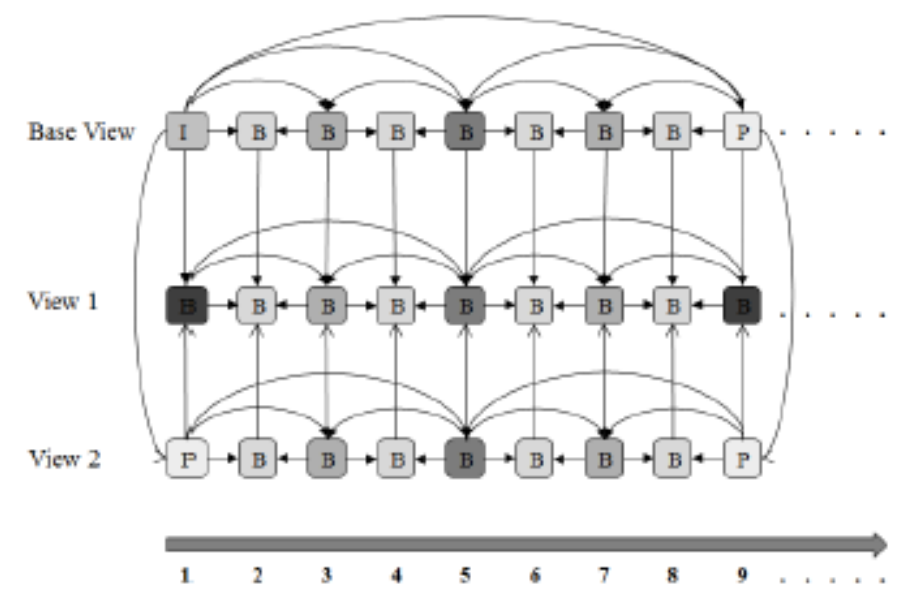

Figure 2. Multiview video GOP 


\subsubsection{Motion Area}

The encoder estimates the motion area of each macroblock (in frame and view) and only the high motion regions are encoded in Intra mode. Figure 3 shows regions of increasing motion for Ballroom, Exit and Vassar sequences. The regions of increasing motion are marked by white circles. Based on these high motion regions, we can select the appropriate AIR technique and decide the refresh strategy.

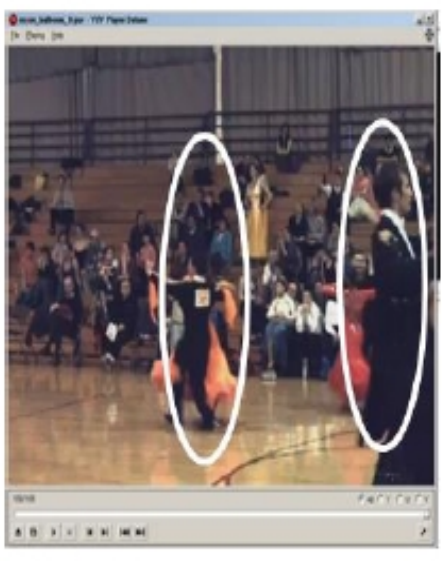

Ballroom

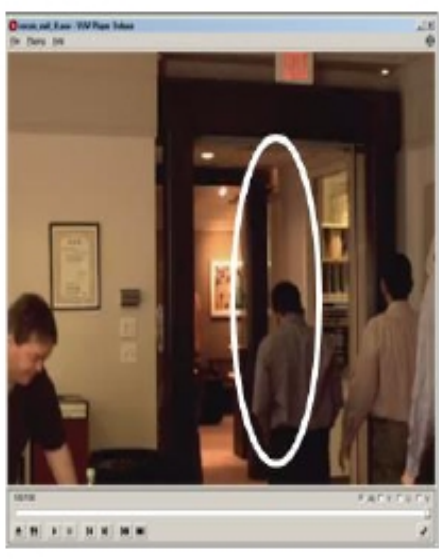

Exit

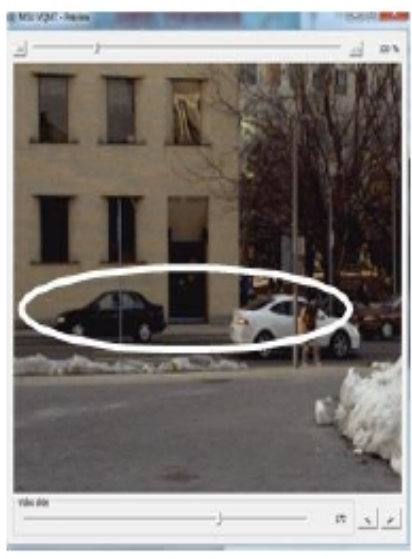

Vassar

Figure 3. The regions of increasing motion

\subsubsection{Refresh Map}

The result of the estimate is recorded as the Refresh Map on a macroblock-by-macroblock basis. An example is presented in Figure 4 (a-f).

\begin{tabular}{|l|l|l|l|l|l|l|l|}
\hline 0 & 0 & 0 & 0 & 0 & 0 & 0 & 0 \\
\hline 0 & 0 & 0 & 0 & 0 & 0 & 0 & 0 \\
\hline 0 & 0 & 0 & 0 & 0 & 0 & 0 & 0 \\
\hline 0 & 0 & 0 & 0 & 0 & 0 & 0 & 0 \\
\hline 0 & 0 & 0 & 0 & 0 & 0 & 0 & 0 \\
\hline 0 & 0 & 0 & 0 & 0 & 0 & 0 & 0 \\
\hline 0 & 0 & 0 & 0 & 0 & 0 & 0 & 0 \\
\hline 0 & 0 & 0 & 0 & 0 & 0 & 0 & 0 \\
\hline
\end{tabular}

a. Opening codes

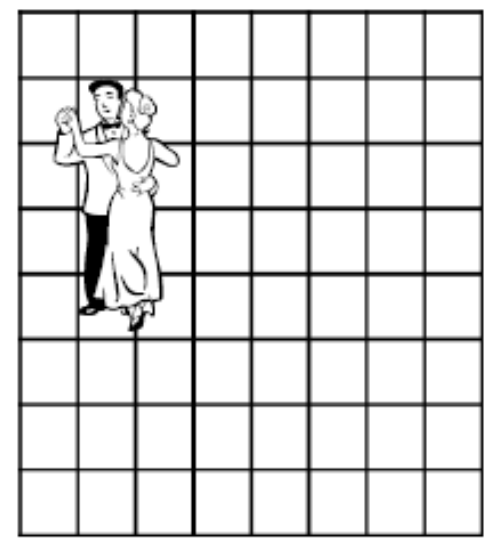

b. Active MB identified

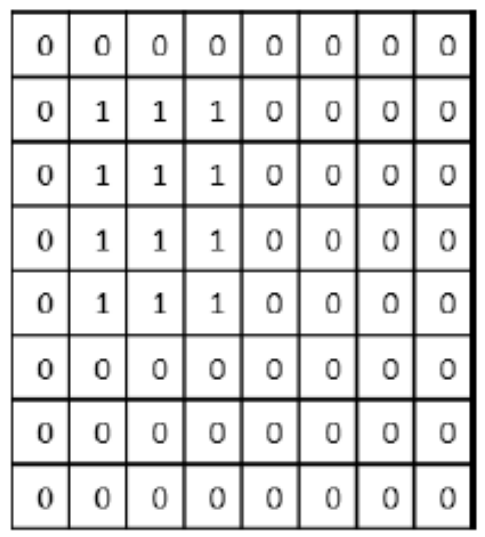

c. First update 


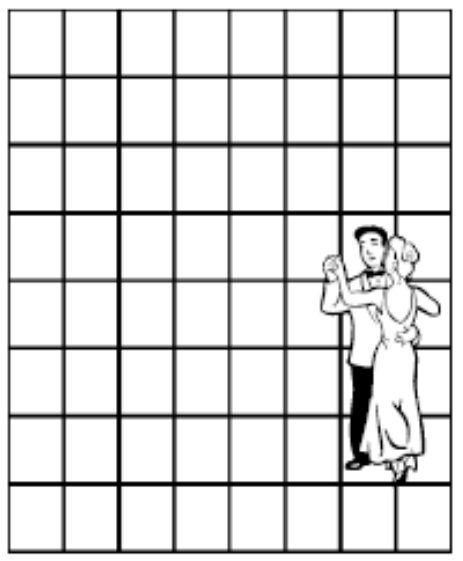

d. Active $\mathrm{MB}$ position

\begin{tabular}{|l|l|l|l|l|l|l|l|}
\hline 0 & 0 & 0 & 0 & 0 & 0 & 0 & 0 \\
\hline 0 & 0 & 0 & 0 & 0 & 0 & 0 & 0 \\
\hline 0 & 0 & 0 & 0 & 0 & 0 & 0 & 0 \\
\hline 0 & 0 & 0 & 0 & 0 & 1 & 1 & 1 \\
\hline 0 & 0 & 0 & 0 & 0 & 1 & 1 & 1 \\
\hline 0 & 0 & 0 & 0 & 0 & 1 & 1 & 1 \\
\hline 0 & 0 & 0 & 0 & 0 & 1 & 1 & 1 \\
\hline 0 & 0 & 0 & 0 & 0 & 0 & 0 & 0 \\
\hline
\end{tabular}

e. Second update

\begin{tabular}{|l|l|l|l|l|l|l|l|}
\hline 1 & 1 & 1 & 1 & 1 & 1 & 1 & 1 \\
\hline 1 & 1 & 1 & 1 & 1 & 1 & 1 & 1 \\
\hline 1 & 1 & 1 & 1 & 1 & 1 & 1 & 1 \\
\hline 1 & 1 & 1 & 1 & 1 & 1 & 1 & 1 \\
\hline 1 & 1 & 1 & 1 & 1 & 1 & 1 & 1 \\
\hline 1 & 1 & 1 & 1 & 1 & 1 & 1 & 1 \\
\hline 1 & 1 & 1 & 1 & 1 & 1 & 1 & 1 \\
\hline 1 & 1 & 1 & 1 & 1 & 1 & 1 & 1 \\
\hline
\end{tabular}

f. Reset

Figure 4. Example of Refresh Map macroblock by macroblock

\subsubsection{Encoding}

The encoder refers to the Refresh Map and decides whether or not to encode the current macroblock in Intra mode. Figure 5 presents an example of a current frame where its macroblocks are feeding macroblocks from other frames in both space and time.

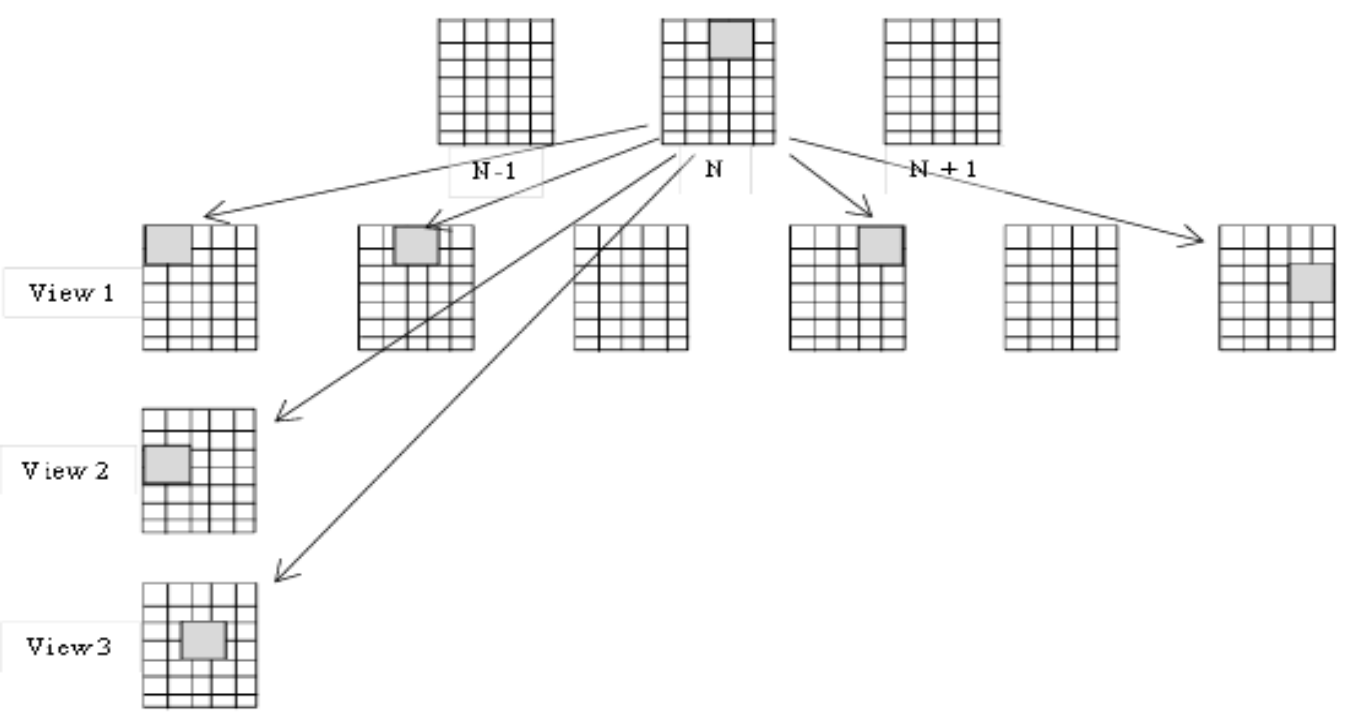

Figure 5. Example of current frame and its dependencies based on a macroblock level

\subsubsection{Sum of Absolute Difference}

Motion estimation is performed by comparing the Sum of Absolute Difference (SAD) against the pre-set threshold value. The proposed scheme exploits the relation between motion and texture within the visual scene. Figure 6 shows the variation in motion over the standard 'Ballroom' 
sequence. In the middle of the sequence, the dancing was at its peak. This period of high motion activity can be detected though the motion values set.

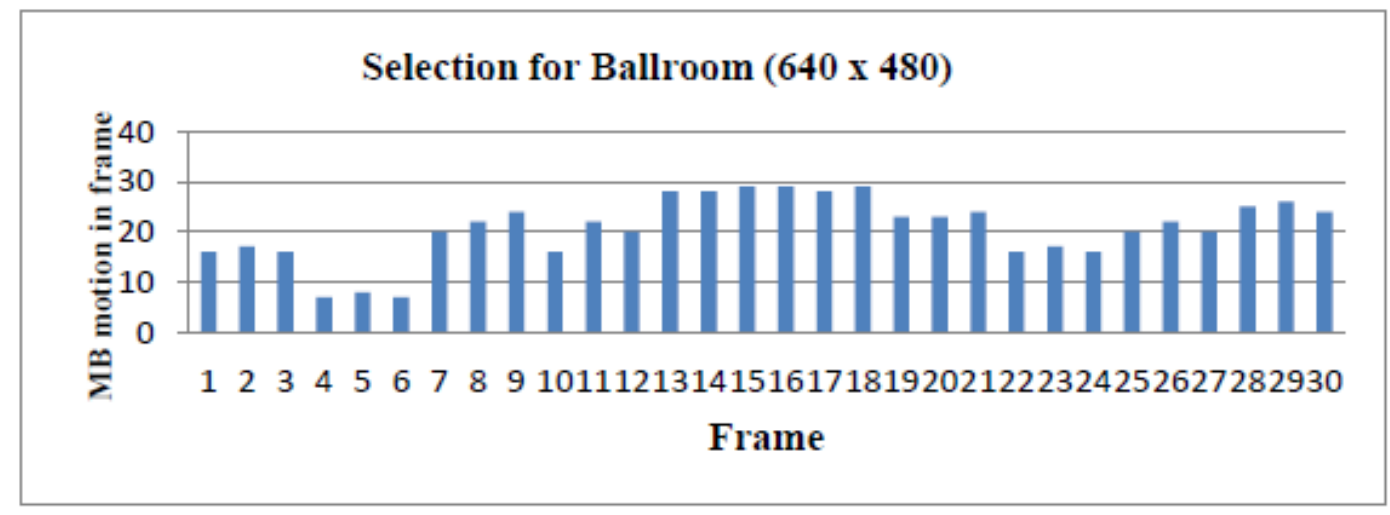

Figure 6. Motion area in Ballroom sequence showing high motion frames (13 to 18)

\subsubsection{Threshold}

If the SAD of the current macroblock is larger than the threshold value, this macroblock is regarded as active, and continues to be in active state until it is encoded in Intra mode at predetermined times. The basic flowchart of SAD tracking is shown in Figure 7. The predetermined values for our test are set, this value is recorded to the Refresh Map.

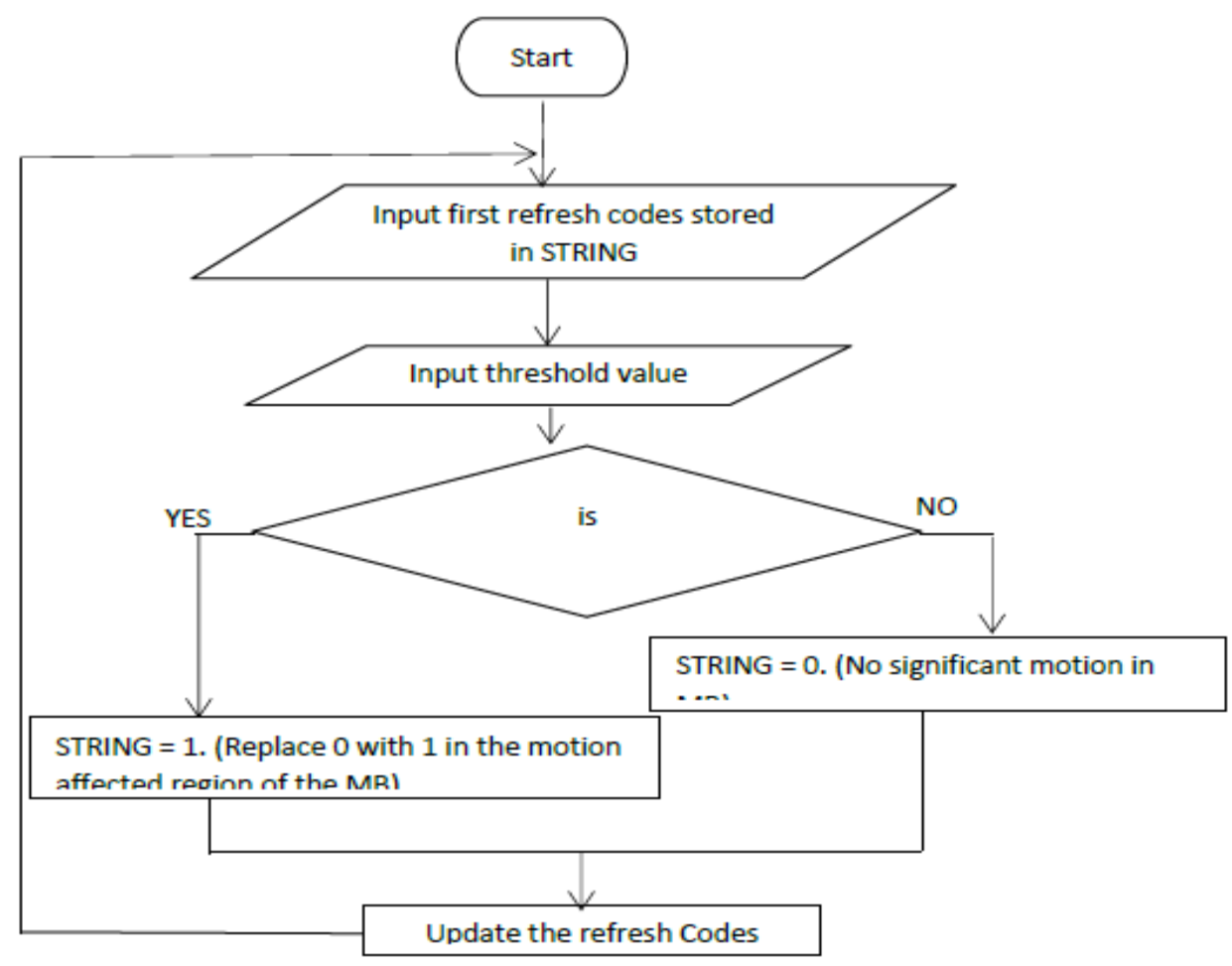

Figure 7. Basic flowchart 


\subsection{Implementation Strategy}

One of the technical features of H.264/AVC is that it supports two types of Intra refresh: (a) IntraDecode Refresh (IDR) and (b) Intra coded macroblock (I-picture) [13]. In the implementation of Intra macroblock refresh using a cyclic method, a block line of Intra coded macroblocks moving from top-to-bottom is employed. The video sequence is updated with intra-coded macroblocks using a pre-defined scan order and selected number of macroblocks per picture. Figure 8 presents an example of the row ordering, starting from row \#1, \#2 up to the last row, then it is reset to first row \#0. Applying this to a sequence of $(640 \mathrm{x} 480)$ spatial resolution, one block equals 40 macroblocks and by refreshing 30 times, the entire frame of 1200 macroblocks is Intra refreshed. Due to the sensitivity of different blocks to channel errors, individual features of the blocks need to be considered.

For the efficient performance of AIR technique, the encoder is required to keep track of the decoder to know which part of the image area was recently refreshed. The encoder would then refresh those active macroblocks, which had an impact on error propagation. Receiver statistics is used to indicate the channel conditions by means of feedback messages. Therefore, a feedback channel is used to link the far-end decoder with the encoder.

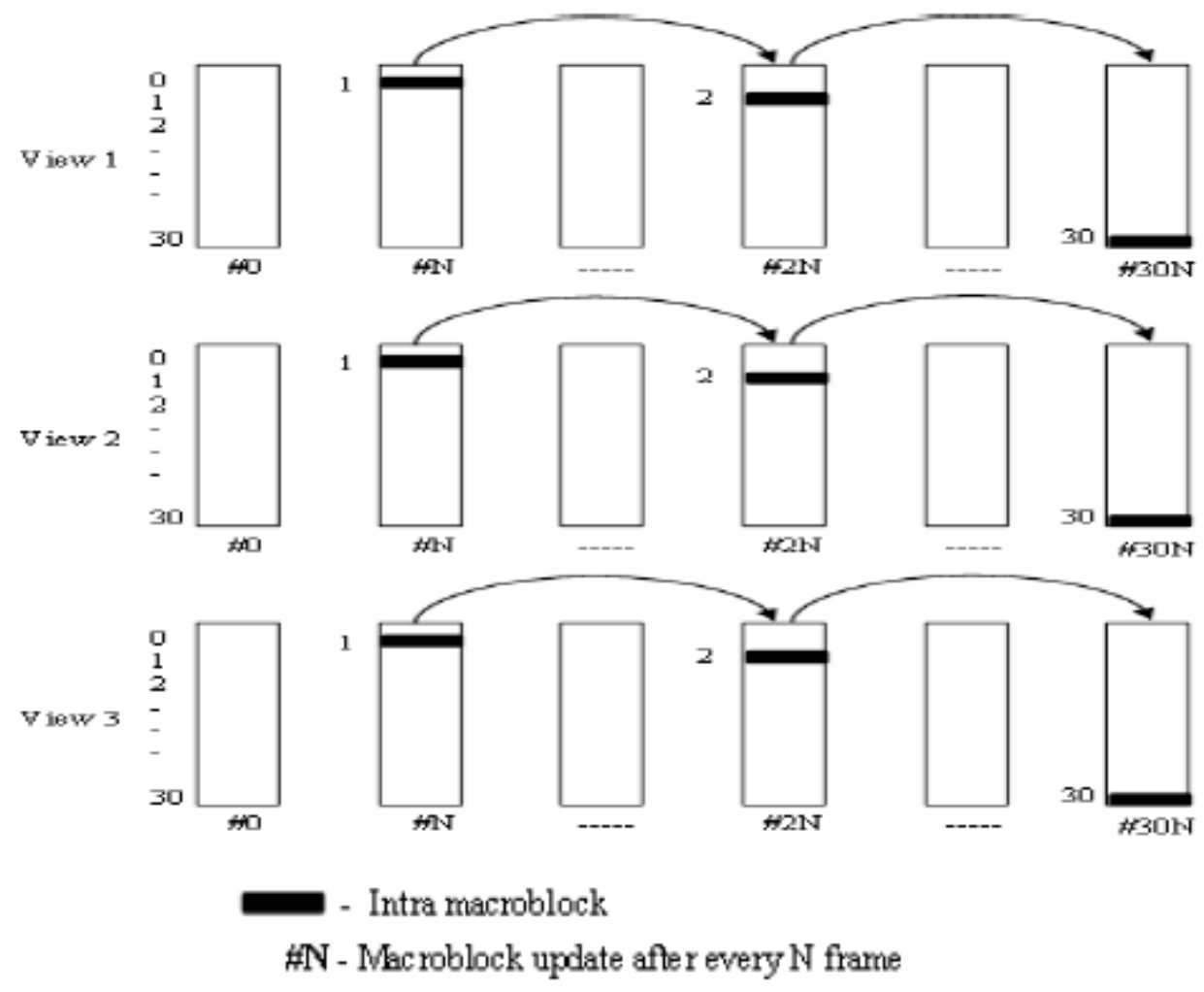

Figure 8. Example of cyclic intra refresh 


\section{EXPERIMENTS}

In this section, we describe the simulations conducted to evalute the performance of AIR in H.264/AVC simulation environment. The results of the test conducted are analysed.

\subsection{Simulation}

The simulations conducted involve running the robust AIR method in H.264/AVC reference JMVC codec [14] [15]. The sequences "Ballroom", "Exit" and "Vassar" were used in our tests. Each of the three views was configured with the parameter in JMVC configuration file in MVC mode. The summary of some important encoding parameters is shown in Table 1. The three camera views for the tests were run serially, but the encoder was made to call each view separately. The MVC bitstream assembler tool was used to assemble individual bitstreams and represent the different views into a single bitstream before decoding. To enable efficient comparison, two different error resilience tools were used, namely flexible macroblock ordering (FMO) represented here as H.264 (A) and arbitrary slice ordering (ASO) represented as H.264 (B). Simulations for both H.264(A) and H.264(B) based on macroblock Intra refresh were also undertaken without the SAD selection process. The data related to the performance of the three different tests schemes was collected.

Table 1. MVV encoding configuration parameters.

\begin{tabular}{|l|l|l|l|}
\hline Serial & Parameter & Specification & remarks \\
\hline 1 & Input file & Ballroom, Exit, Vassar & \\
\hline 2 & Resolution & $640 \times 480$ & \\
\hline 3 & Frame rate & $25 \mathrm{~Hz}$ & \\
\hline 4 & Frame encoded & 200 & \\
\hline 5 & Quantization parameter (QP) & $25,27,29,31,33$ & \\
\hline 6 & Means macroblocks & 1 & Adaptively selected \\
\hline 7 & GOP size & 12 & \\
\hline 8 & Intra period & 12 & \\
\hline 9 & Number of reference frame & 2 & I and P \\
\hline 10 & Number of views & 3 & \\
\hline
\end{tabular}

\subsection{Test Results}

In the first set of the experiments, our goal is to increase the robustness of the compressed bitstream to channel errors. Therefore, the performance between the original and reconstructed sequences was measured in terms of the frames Peak Signal to Noise Ratio (PSNR) which is the standard objective quality metric used for video objective quality assessment. We compare the three coding schemes in both error free and error prone environments. Figure 9 presents the PSNR performance of the "Ballroom" sequence encoded at various GOP refresh rates. It is clear to observe that the performance of the proposed AIR scheme is much better than that of the other two schemes in all the refresh rates considered. 
International Journal of Computer Science, Engineering and Applications (IJCSEA) Vol.4, No.6, December 2014

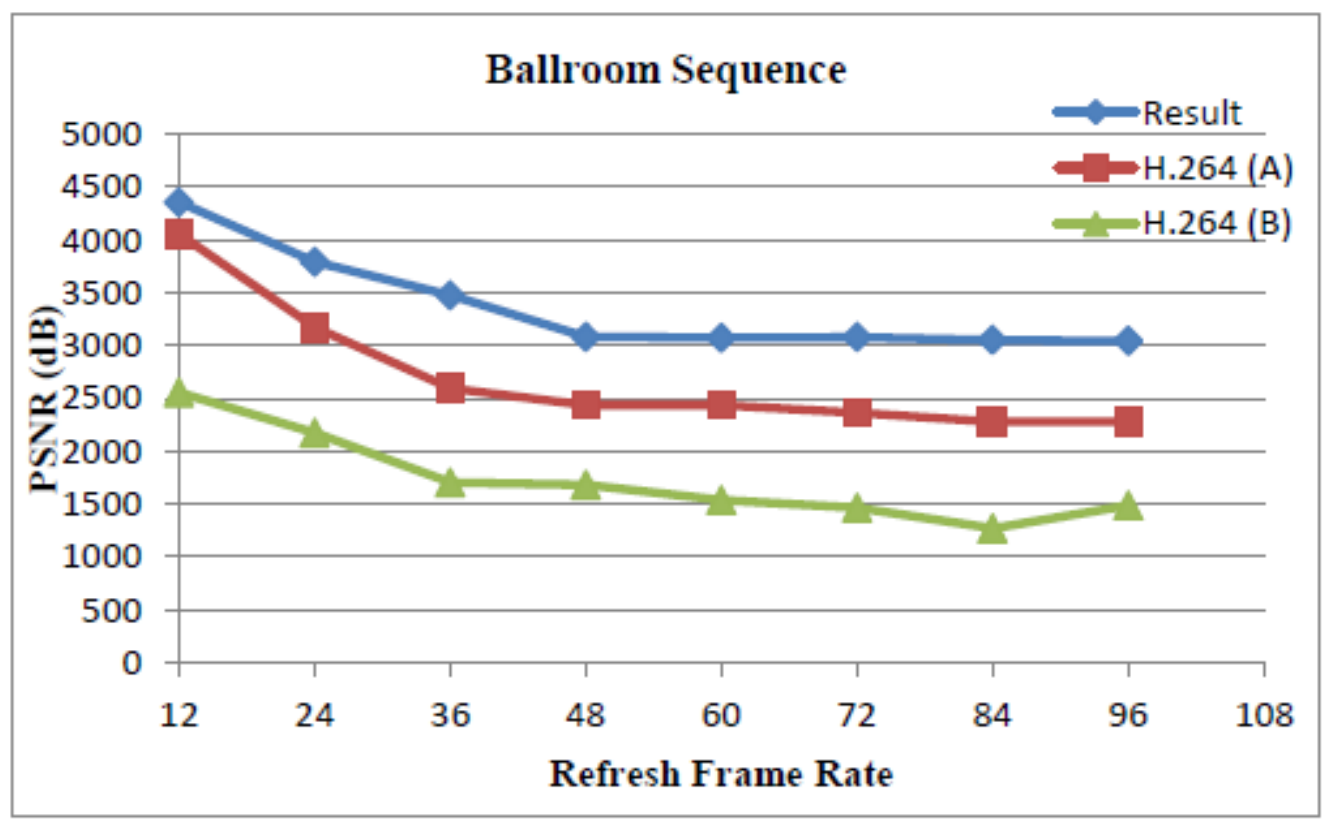

Figure 9. Performance of sequence Ballroom compared with two H.264 schemes

Similarly, Figure 10 shows the performance when "Exit" sequence was used. It is clear that increasing the frequency of Intra refreshing in the GOP presents better video quality under AIR conditions. It can be observed that adaptive Intra refresh coding alone yields better results than error concealment alone. Combining cyclic adaptive intra refresh coding and error concealment at the decoder shows an overall gain of up to $4 \mathrm{~dB}$.

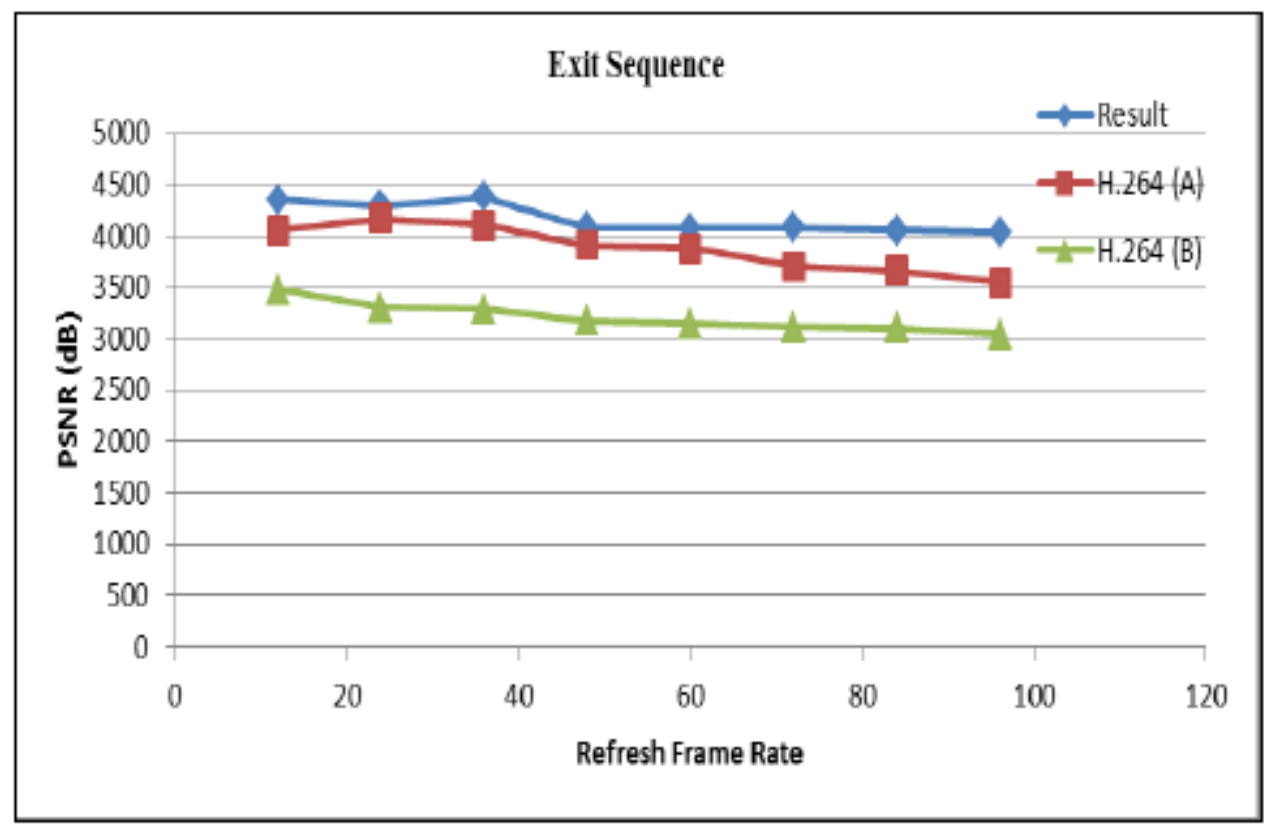

10. Performance of sequence Exit compared with two H.264 schemes 
The finding of this research is consistent with findings of past studies [3]-[6]-[7]-[9], which indicates that when an error occurs in one view of $\mathrm{MVC}$, this error propagates in time and to the other views causing significant perceptual quality degradation in the decoded videos. It was confirmed that errors propagates due to the temporal/inter-view prediction structure utilized by the MVC scheme. The gains obtained from AIR error resilience techniques on the base view that does not contain any inter-view correlations, are all comparable to those reported in [7] and [9]. Subjective results achieved with the "Ballroom", "Exit" and "Vassar" sequences are presented in Figure 11 below.

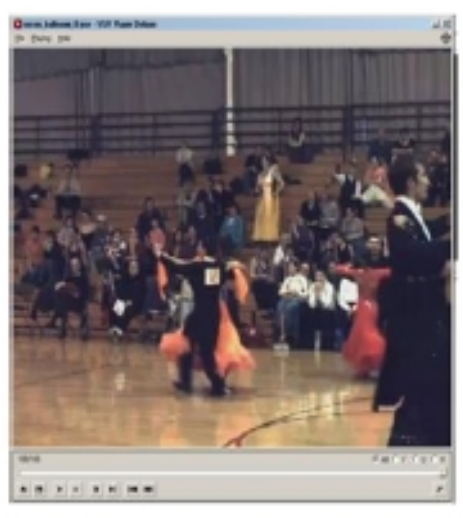

a. AIR scheme

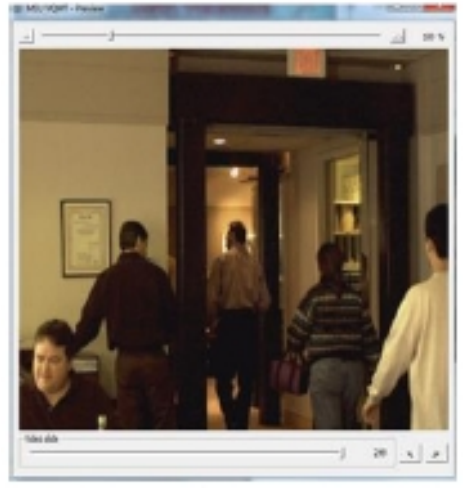

a. AIR scheme

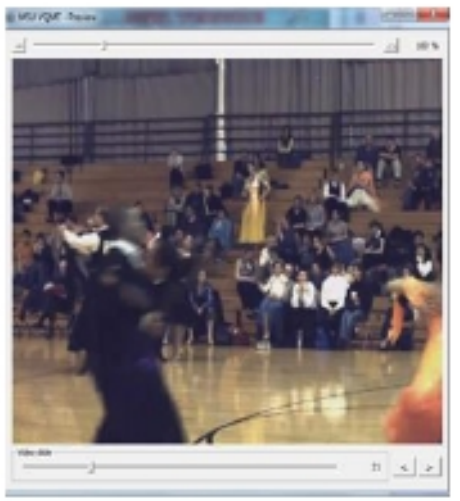

b. H.264 (A) scheme

Ballroom

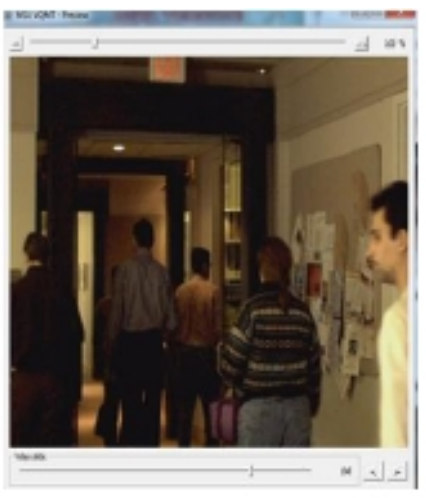

b. H.264 (A) scheme

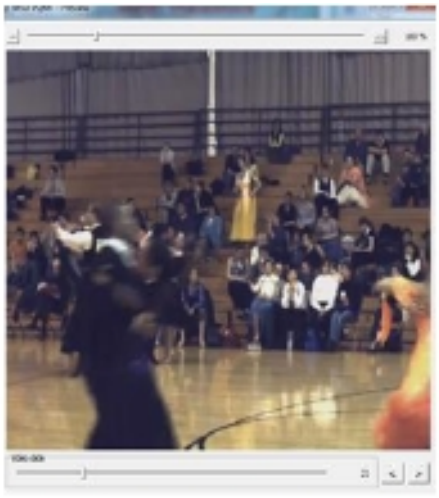

c. H.264 (B) scheme

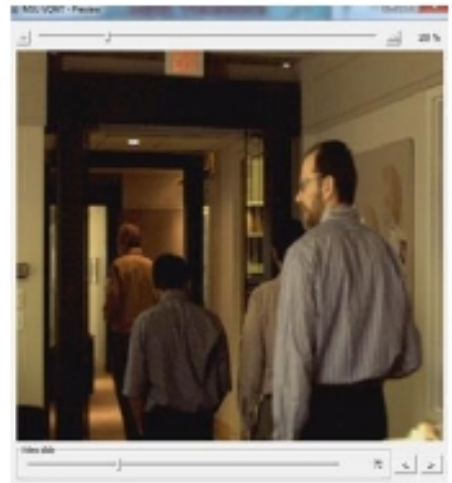

c. H.264 (B) scheme

Exit 


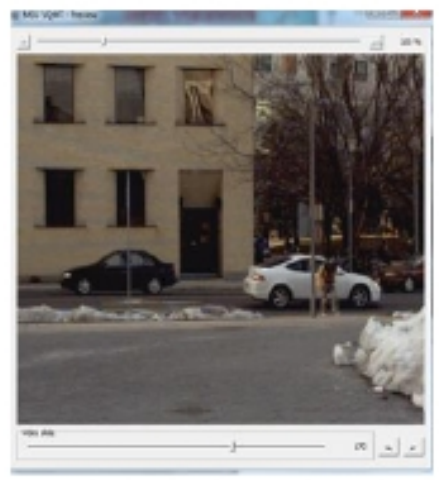

a. AIR scheme

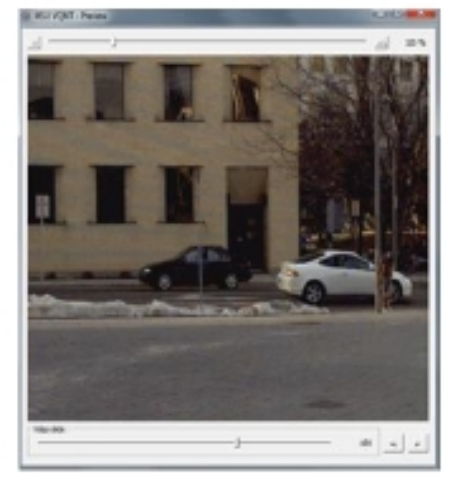

b. H.264 (A) scheme

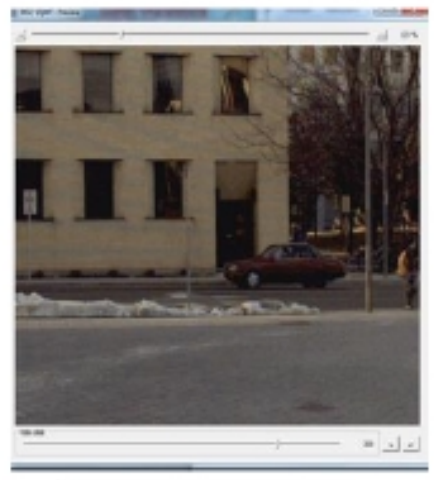

c. H.264 (B) scheme

Vassar

11. Subjective performance of Ballroom, Exit and Vassar

\section{CONCLUSIONS}

This paper presented results of AIR scheme for MVC in H.264/AVC. A cyclic Intra refresh method in H.264/AVC is introduced, taking into account the features of each individual macroblock. To validate bitstream recovery from errors, two additional Intra coded H.264 schemes were introduced. Simulations are conducted using H.264/AVC for multiview video. For the purpose of comparison, this paper has employed both objective and subjective video quality assessment. Results show the proposed solution presents a superior perceptual video quality to the method used in the H.264/AVC reference codec.

\section{REFERENCES}

[1] B. Briscoe, "Non-conventional armed violence and non-state actors: challenges for mediation and humanitarian action," 2013.

[2] A. H. Sadka, Compressed Video Communications, published by J. Wiley, 2002.

[3] J. Jiang, B. Guo and W. Mo, "Efficient intra refresh using motion affected region tracking for surveillance video over error prone networks," in Intelligent Systems Design and Applications, 2008. ISDA'08. Eighth International Conference on, 2008, pp. 242-246.

[4] Q. Chen, Z. Chen, X. Gu and C. Wang, "Attention-based adaptive intra refresh for error-prone video transmission," Communications Magazine, IEEE, vol. 45, pp. 52-60, 2007.

[5] G. Côté and F. Kossentini, "Optimal intra coding of blocks for robust video communication over the Internet," Signal Process Image Commun, vol. 15, pp. 25-34, 1999.

[6] Y. Zhou and Y. Chen, "Error-resilient video coding of H. 264/AVC based on network-adaptive intra refresh and reference selection refresh," Optical Engineering, vol. 49, pp. 077401-077401-11, 2010.

[7] Y. Wang, M. M. Hannuksela and M. Gabbouj, "Error-robust inter/intra macroblock mode selection using isolated regions," Proc.13th Packet Video Wksp, 2003.

[8] G. J. Sullivan and T. Wiegand, "Rate-distortion optimization for video compression," Signal Processing Magazine, IEEE, vol. 15, pp. 74-90, 1998.

[9] X. Wang, C. Kodikara, A. Sadka and A. Kondoz, "Robust GOB intra refresh scheme for H. 264/AVC video over UMTS," in 3G and Beyond, 2005 6th IEE International Conference on, 2005, pp. 1-4.

[10] L. Al-Jobouri, M. Fleury and M. Ghanbari, "Intra-Refresh Provision for Data-Partitioned H. 264 Video Streaming over WiMAX," Consumer Electronics Times. 
International Journal of Computer Science, Engineering and Applications (IJCSEA) Vol.4, No.6, December 2014

[11] I. Draft, "recommendation and final draft international standard of joint video specification (ITU-T Rec. H. 264| ISO/IEC 14496-10 AVC)," Joint Video Team (JVT) of ISO/IEC MPEG and ITU-T VCEG, JVTG050, vol. 33, 2003.

[12] S. Worrall, A. Sadka, A. Kondoz and P. Sweeney, "Motion adaptive intra refresh for MPEG-4," Electron. Lett., vol. 36, pp. 1924-1925, 2000.

[13] Y. J. Liang, K. El-Maleh and S. Manjunath, "Upfront intra-refresh decision for low-complexity wireless video telephony," in Circuits and Systems, 2006. ISCAS 2006. Proceedings. 2006 IEEE International Symposium on, 2006, pp. 4 pp.

[14] J. Xu and Z. Wu, "Joint adaptive intra refreshment and unequally error protection algorithms for robust transmission of H. 264/AVC video," in Multimedia and Expo, 2006 IEEE International Conference on, 2006, pp. 693-696.

[15] P. Chang and T. Lee, "Precise and fast error tracking for error-resilient transmission of H. 263 video," Circuits and Systems for Video Technology, IEEE Transactions on, vol. 10, pp. 600-607, 2000. 